\title{
Two tests of a neural attention hypothesis for auditory psychophysics
}

\author{
R. DUNCAN LUCE and DAVID M. GREEN \\ Laboratory of Psychophysics, Harvard University, Cambridge, Massachusetts 02138
}

\begin{abstract}
A theory of neural attention is presented, and it is argued that intensity attention bands and critical bands of frequency are simply two manifestations of it. This suggests studying it by taking observations on the one variable while manipulating the other over a large range. The first experiment is a conventional magnitude estimate experiment, except that on any trial one of two different signal frequencies are presented at random. Using as measures relative variability in the ratio of successive responses and the correlation between them, it appears that a large change in intensity at the same frequency on successive trials is equivalent to a large change in frequency on those trials. The second experiment is a simple two-frequency identification experiment, except that the stimulus intensity varies randomly from trial to trial according to a predetermined distribution in which there is a tight cluster of intensity as well as two outliers. Identification is better when the stimulus intensity is chosen from the cluster than when it is an outlier, which is what one would expect if the attention band is located to encompass the cluster.
\end{abstract}

Earlier, we suggested the possible existence of an auditory attention mechanism for intensity (Green \& Luce, 1974). It takes the form of a band of intensities, of variable location and possibly whose width varies with location, such that signals falling within the band are attended to more closely than those falling outside it. We interpret "attended to more closely" to mean that the neural representation of a signal falling within the attended band has a sample size that is substantially larger than when the same signal falls in the unattended region. This means that parameters of the signal, such as its intensity and frequency, can be estimated with greater precision when the signal falls within the band than when it is outside. The location of the band is not fixed, but, rather, varies in time in a fashion not fully understood. As will be discussed more fully below, we believe that a central process determines the differential monitoring of the peripheral neurons.

This hypothesis provides a simple explanation of several otherwise perplexing empirical phenomena which appear when a wide range of intensity is used, as in magnitude estimation and production, category methods, and absolute identification. The result which first led to the idea of such a band is the fact that the relative variability or coefficient of variation (standard deviation divided by the mean) of the ratio of magnitude estimation responses on

This work was supported in part by National Science Foundation grants to Harvard University. An earlier draft of this paper was commented on by John Baird, N. I. Durlach, Marilyn L, Shaw, and Daniel Weber. We appreciate their helpful comments. successive trials is smaller by a factor of about $1 / 3$ when the two corresponding signals are physically close than when they are widely separated. Baird (1970) noted the existence of this phenomenon in several modalities and referred to it as a $\mathrm{V}$ pattern. We have studied it for auditory intensity in both magnitude estimation (Green \& Luce, 1974) and magnitude production as well as estimation (Green; Luce, \& Duncan, 1977). A second, and we now suspect more basic, phenomenon is seen by studying the correlation of $\log R_{n}$ vs. $\log R_{n-1}$, where $R_{n}$ is the response on trial $n$, as a function of signal separation. These correlations exhibit a triangular pattern, with a high value of about .85 when the signals are identical and a value that drops to 0 for large signal separations. This correlation pattern holds for both estimation and production data (Green et al., 1977; Jesteadt, Luce, \& Green, 1977). When we turn to absolute identification, there are two, probably closely related, phenomena accounted for by the attention band. The first is the classical capacity effect, identified by Miller (1956) as the magical number $7 \pm 2$, which says that once one exceeds about seven equally spaced signals, successive ones become confused despite the fact that when presented in pairs they are perfectly identifiable (Garner, 1953). Similarly, if the number of signals is held fixed and their range is increased, the rate of improvement of performance falls off rapidly once the range is greater than $20 \mathrm{~dB}$ (Braida \& Durlach, 1972). Luce, Green, and Weber (1976) have shown how both of these results follow quite directly from the attention band hypothesis. The basic idea is that, in experiments with a wide range of signal 
intensities, the attention band can only cover some of the intensities and hence a substantial fraction of the signals are represented by a small sample size, whereas in any narrow range experiment the band can be and is located so that all signals receive the larger sample size.

This paper reports two additional experiments designed to explore other implications of the basic idea that the central nervous system cannot fully monitor all the peripheral neurons.

\section{PROPERTIES OF ATTENTION BANDS}

The key properties of such attention bands are believed to be the following:

(1) The neural sample size is larger by about an order of magnitude when the signal lies in the band than when it lies outside it. Assuming that the psychological representation of the signal arises as some amalgamation, perhaps an average, of different representations obtained from individual nerve fibers, a signal falling within the band has an internal representation with a standard deviation about $1 / 3$ of that produced when the same signal falls outside the band because of the difference in sample size. Attention, according to this view, is equated with the idea of accumulating a larger sample from which various aspects of the signal can be estimated.

(2) The attention band can be located anywhere in the intensity range. Thus far, we do not have much experimental control over its location, but the evidence suggests that most observers exhibit at least two tendencies: one is to locate the band either where the last signal was or where they expect the next one to be-the data tend to confound the two, but where they have not they tend to favor the latter hypothesis (Green \& Luce, 1974); the other is to locate it at the most intense signal in the set of signals being used (Berliner \& Durlach, 1973; Weber, Green, \& Luce, 1977).

(3) Because we do not know with any certainty how to control the location of attention band of intensity, it has not yet been possible to study how the width of the band varies as a function of its location along the intensity variable. Indeed, for all we know, it may be possible for the observer to divide his total attentional capacity into several noncontiguous regions. Because we do not know how to study its local behavior, we have fit data on the assumption that the width (in decibels) is constant and independent of intensity. On that assumption, we get estimates for the average width of between 10 and $20 \mathrm{~dB}$.

(4) When two successive signals are sufficiently close so that they can be encompassed by a single band, it appears that observers can "calculate" the ratio of the two representations and preserve that ratio in their responses, as they are often instructed to do in magnitude estimation and production experiments. We have called this behavioral property the response ratio hypothesis (Luce \& Green, 1974). However, when signals are so far apart that they are not within a single band, the response to the second of two signals does not appear to be influenced either by the previous signal or by the previous response (Green et al., 1977; Jesteadt et al., 1977). So far as we know, this unexpected feature of the band is totally independent of the differential sample size, and we do not have any very clear intuitions as to why it occurs, how else it may evidence itself, or why it is useful. Clearly it bears further investigation.

\section{RELATION TO CRITICAL BANDS FOR FREQUENCY}

The concept of attention bands for intensity parallels that of critical bands for frequency. Both are interpreted as forms of selective attention, and they reflect the limited capacity of the nervous system to process sensory information. We wish to suggest the stronger view that they are, in fact, the intensity and frequency manifestations of a single neural process.

The usual view of the critical band is that it represents a narrow region on the basilar membrane. The central nervous system somehow measures the total activity (spike count) for this small set of fibers and largely ignores activity arising from more distant regions. The mechanical filtering of the cochlea precedes this neural counter; hence the critical band is essentially a mechanical filter and the spike counter measures the amplitude at the output of the filter. This view is certainly consistent with the traditional resonance theory of hearing.

If one accepts the idea that the peripheral fibers have a relatively restricted dynamic range and that the range of absolute sensitivity of neighboring fibers is nearly the same, then the classical view has trouble for all but threshold signals. The difficulty is that a moderately intense sinusoidal signal will saturate an appreciable band of peripheral fibers located along the basilar membrane and that, hence, only gross information about the frequency or intensity of the sinusoid is available. Of course, not all fibers are saturated; there are fibers located on each side of this band that are not saturated yet are stimulated above their resting rates. These fibers are not maximally sensitive to the frequency of the signal but rather to frequencies above or below the signal frequency. Acceptance of these views-which are consistent with our present understanding of peripheral auditory function-leads us to consider rather different views of the neural mechanism 
responsible for selective attention, either in frequency or intensity. We develop this alternative view.

The tuning curve of an auditory fiber is defined as those combinations of sinusoidal frequency (f) and intensity (I) that just stimulate the fiber above its resting or spontaneous level of activity. In a diagram of $\log I$ vs. $\log \mathrm{f}$, the most sensitive part of the tuning curve is roughly $V$-shaped, with its right limb considerably steeper than the left limb. In addition, at frequencies below those of the left limb, there is a region which is roughly constant in sensitivity and about 40 to $50 \mathrm{~dB}$ less sensitive than the tip of the $V$. The tip of the $V$, the frequency at which the fiber is most responsive, is called the best or characteristic frequency of the fiber. As far as we know, almost all fibers of the same characteristic frequency have similar $( \pm 10 \mathrm{~dB})$ thresholds (Evans, 1975; Kiang, 1968). As one raises the intensity of the stimulus, ultimately the fiber's rate of firing deviates from its resting level and increases to its maximal level. At any frequency, the range of intensities needed to move it from resting to maximal level is only about 20-30 dB (Galambos \& Davis, 1943; Kiang, 1965). We refer to fibers that are firing at rates somewhere between the resting and the maximal rates as differentially active-differential because they change their response to small changes in either frequency or intensity of the signal.

It is clear that sinusoids near threshold activate only those fibers whose characteristic frequency is close to the signal frequency. This is not true for suprathreshold signals. Consider a sinusoid of moderate (50-80 dB) intensity. Then two distinct groups of fibers are differentially active: The one has characteristic frequencies less than the signal frequency, and hence the signal is activating the right limb of their tuning curves. The other has characteristic frequencies greater than the signal frequency, and hence the signal is activating the left limb of their tuning curves. The greater the intensity, the wider the frequency separation between these two groups of fibers.

If we study the spike trains that are generated by signals with frequencies less than $2,000 \mathrm{~Hz}$ in fibers that are differentially active, we find both intensity and frequency information encoded in the pulse train. To be specific, the distribution of times between successive spikes-the interval histogramexhibits a multimodal pattern, with the modes spaced at integral multiples of the period of the signal, and the parameter of the geometric decay of the modes increases with intensity (Rose, Brugge, Anderson, \& Hind, 1967). Because of the limited dynamic range of the fibers, it is clear that full intensity information cannot be provided just by the differentially active fibers any more than by those fibers whose characteristic frequency is the same as the signal, which are largely saturated. Indeed, since the total number of saturated fibers increases with intensity, they provide a crude measure of intensity, to which the geometric parameters of the differentially active ones provide a finer vernier.

Many fibers are substantially identical, i.e., they have the same characteristic frequency. These similar fibers encode the same information, but presumably do so independently. Thus, averaging in some way over the differentially active fibers permits the system to increase the total sample size from which inferences are drawn about the signal without greatly increasing the time required. This suggests thinking of the 30,000 peripheral fibers as partitioned into a number of equivalence classes.

Our neural attention hypothesis is that, at any instant, one of these equivalence classes is fully monitored by the central nervous system, and all of the others are represented by a sample which, according to current estimates, is about $1 / 10$ of the fibers in the class. This reduces the computational burden on the nervous system by almost a factor of 10 while allowing it to retain partial information about all frequencies and intensities. Which class is monitored fully can vary from situation to situation or instant to instant, and we do not understand very well what controls the choice. According to this hypothesis, then, the attention band for intensity and critical band for frequency reflect, in effect, the height and width, respectively, of the region of differential activity within the fibers of the fully monitored class.

This theoretical background provides the motivation for both of our experiments. The first takes advantage of the fact that large changes in frequency should suffice to activate a different class of fibers and so affords the opportunity of bypassing the attention mechanism, even if responses are only to intensity. The second takes advantage of the fact that the same is true for large changes in intensity, even if responses are only to frequency.

\section{EXPERIMENT 1 TWO-FREQUENCY MAGNITUDE ESTIMATION}

We know that, with a signal of fixed frequency, two aspects of performance in a magnitude estimation experiment-the coefficient of variation of the ratio of successive responses and the correlation between them-vary systematically with the difference in intensity between successive signals. We interpret this result to mean that the attention band tends to be located at the intensity of the last signal; hence, for the reasons discussed above, we obtain measurable differences, depending on the intensity differences of successive signals. If the sample size of the representation of a signal depends on partic- 
ular groups of auditory fibers being monitored, then one way to disrupt the process would be to change the frequency of the signal on successive trials. If the attention band is located at those fibers differentially activated by the previous signal, then changing the frequency will, in general, cause the next signal to lie outside the band whether or not the intensity changes a lot or a little. In effect, the change in frequency should have an impact on the representation equivalent to a very large change in intensity at the same signal frequency.

\section{Procedure}

The signals were 1,000 - and $4,000-\mathrm{Hz}$ tones of $500-\mathrm{msec}$ duration, presented binaurally, in quiet, via TDH-39 headphones to observers who were tested in single-walled sound-treated chambers (IAC-402 A). At each frequency, the 21 signal intensities ranged from 45 to $85 \mathrm{~dB}$ SPL in 2-dB steps. The observers were instructed to assign numbers to the successive tones so that the ratios of the numbers were the same as the ratios of the successive loudnesses. Practice, which ranged from about 500 trials for E.N. and C.K. to more than 2,000 trials for the other three observers, was completed before the present data were taken. During the practice period, the data were monitored and observers were encouraged to be consistent; the group with the greater practice was explicitly instructed to try to produce responses such that a change of $10 \mathrm{~dB}$ in intensity produced a change of a factor of 2 in their numbers (a slope of $1 / 3$ in the psychophysical function). This was accomplished as follows: After each run of 100 trials, the individual slopes were measured and the observers encouraged to increase or decrease their range of numbers. After several such blocks, the observers could give data that fell almost exactly along a slope of $1 / 3$.

Responses were obtained either from a Video Systems Terminal (essentially a typewriter keyboard and a small TV screen) or a special response box containing 16 keys (digits $0-9$, a decimal point, and five control functions) and a small LED display (five possible characters). In either case, the observer's response appeared in the display as it was entered, and mistakes could be cleared and another number entered. When the observer was satisfied with the response, it was recorded by the computer (PDP-15) and the next trial was initiated. The procedure was self-paced, with the response to the signal initiating the next trial. Runs consisted of 100 trials, with observers completing between four and six runs separated by rest periods in a $2-h$ session. Sometimes the observers observed in pairs (thereby yoking their pace) and sometimes separately. Approximately 3,000 observations were taken from each observer. They were paid $\$ 3 / \mathrm{h}$.

\section{Results}

Figure 1 presents the average magnitude estimate vs. signal intensity for each frequency and all the observers. The data at each frequency form approximate power functions of intensity with an exponent which is characteristic of the observer. The data for observers A.N. and M.W. fall almost exactly on a slope of $1 / 3$, as is consistent with their explicit instructions, but those of N.R., who was also so instructed, give a smaller exponent, as do those of E.N. and C.K., who received no special instructions.

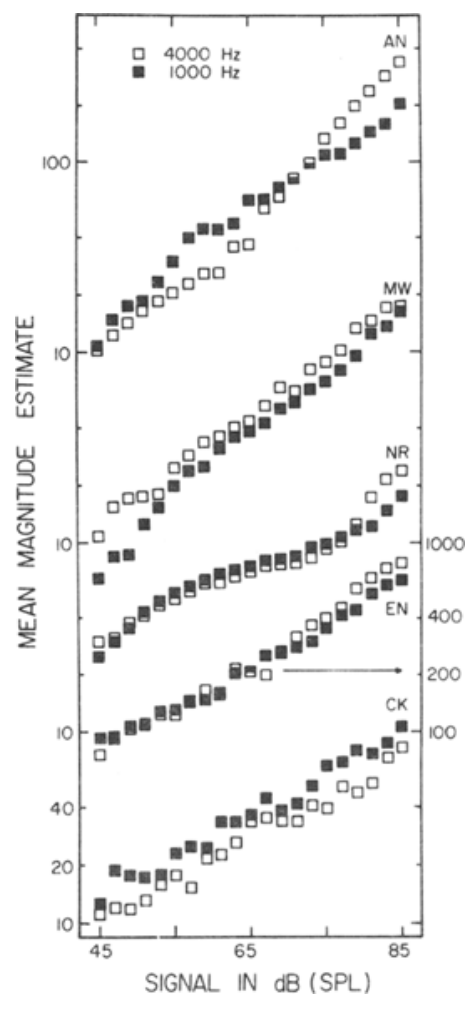

Figure 1. Mean magnitude estimate vs. signal sound pressure level ( $\mathrm{dB}$ re $\mathbf{0 . 0 0 0 2}$ dynes $/ \mathrm{cm}^{2}$ ) for the two frequencies. For four observers, the left ordinate is the correct scale, with an average response of about $\mathbf{1 0 - 2 5}$ for the weakest stimulus. For observer E.N., read the right ordinate, as indicated by the arrow, i.e., the weakest stimulus yielded a mean of about 100 .

More pertinent to the attention band hypothesis is the plot of the coefficient of variation of the ratio of successive responses as a function of the signal separation in decibels on the successive trials (Figure 2). The coefficient of variation is computed separately for those pairs of trials where the signal frequency changed (solid symbols) and where it was the same (open symbols) on successive trials. According to the hypothesis that the attention band is placed on fibers associated with the frequency that occurred on the previous trial, we should see the $V$ pattern for the open symbols and not for the solid symbols. The latter should be constant, independent of intensity, at a value approximately equal to that of a large change in intensity when the frequency remains unchanged. Observers A.N., M.W., and E.N. exhibit this pattern almost exactly. Observer N.R. exhibits a $\mathrm{V}$ pattern on both types of trials, although it is somewhat attenuated when the frequency changes. This performance is not consistent with the predictions; rather, it suggests a pattern of switching sometimes to the other frequency, but maintaining the 


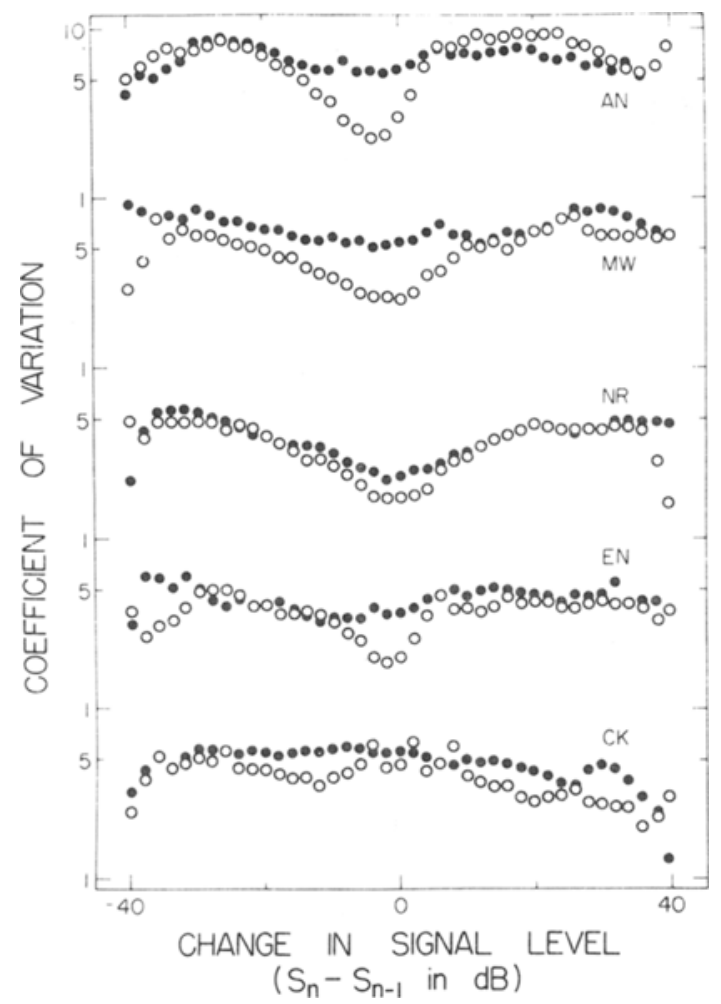

Figure 2. Coefficient of variation computed from the ratio $R_{n} / R_{n-1}$ as a function of the stimulus change in decibels. Solid points represent those successive trials where the signal frequency changed; open points represent the successive trials with no change in frequency.

same intensity. Observer C.K. shows almost no change in the coefficient of variation for any condition, i.e., no evidence of an attention band in any condition. It should be noted that the value of the coefficient of variation for C.K. is about that of the higher level for the other observers. This suggests that he located the attention band in a haphazard fashion.

The second aspect of the data analysis pertinent to the attention band hypothesis is the pattern of correlation between successive responses. Figure 3 shows these correlations ${ }^{1}$ as a function of the change in signal level in decibels for all observers except C.K. The open symbol represents the average correlation when the frequency did not change on successive trials. These results are very similar to those found previously by Jesteadt et al. (1977) and Green et al. (1977), who used a single frequency on all trials. The results for those trials where a change in frequency occurred (solid symbol) show a similar pattern except that the maximum of the correlation is about .5 rather than .8 . The vertical lines about each symbol represent the standard deviation com- puted over the four observers and clearly indicate that the individual observers follow the same trends. These correlations are based on as many as 850 observations for changes of $0 \mathrm{~dB}$ and on as few as 300 observations for changes as large as $33 \mathrm{~dB}$.

Figure 4 is the corresponding plot for the aberrant observer, C.K. The correlation for the no-change trials is uniformly high, about .7 , and the trials in which the frequency changes show a lower level, about .4.

\section{Discussion}

Looking at the coefficient of variation data first, the experiment is a partial success and is supportive of the hypothesis which led to its design. Three observers, A.N., M.W., and E.N., appear much of the time to have held the attention band at both the intensity and frequency of the last signal. One observer, N.R., appears to have done that part of the time, but also to have shifted it some of the time to the other frequency at the same intensity, and the fifth observer's data (C.K.'s) are consistent with a randomized location of the band.

When we turn to the correlation data, we again find a major difference between four of the observers and the fifth one. The first four observers are substantially alike: when the frequency does not change, they exhibit the triangular pattern we have seen before; and when the frequency changes, they exhibit a somewhat attenuated version of it. The fifth observer is quite different: his correlation is constant,

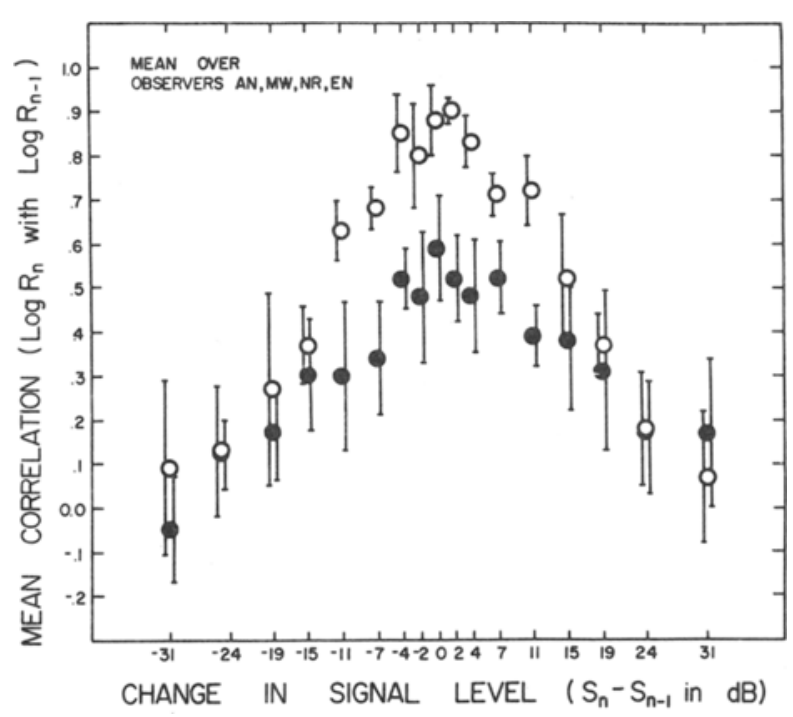

Figure 3. Average correlation of $\log R_{n}$ and $\log R_{-1}$ for a given stimulus change in decibels for the four subjects with similar data. See Footnote 1 for an explanation of how averages were obtained. 


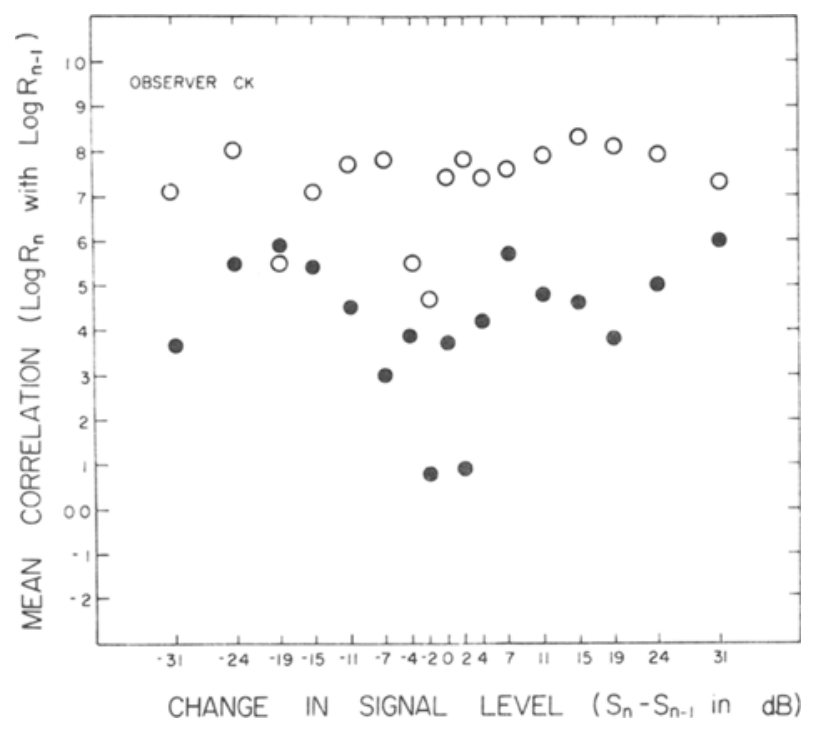

Figure 4. Same as Figure 3 for subject C.K.

not triangular, and it is comparatively high when the frequency does not change and moderate when it does.

There are two questions to be considered. First, does one of these two phenomena-the pattern of the coefficient of variation or that of the correlationderive from the other? In discussions with John Baird, we have concluded that when the correlation pattern is triangular, the pattern of variability is a necessary consequence of it. The argument is this. Consider two regions of the correlation surface: when $S_{n}=S_{n-1}$ and when $S_{n}$ is far from $S_{n-1}$. The distribution of responses on trial $n-1$ will be the same in these two cases. However, when $S_{n}$ $=S_{n-1}$, the scatter diagrams of $\log R_{n}$ vs. $\log R_{n-1}$ will be long and narrow along the line of slope 1 , and so the variability along the line of slope -1 will necessarily be small. If, however, $S_{n} \gg S_{n-1}$ or $S_{n} \ll<S_{n-1}$, there is no correlation between $\log R_{n}$ and $\log R_{n-1}$, and so the variability of $\log R_{n}$ must be the same as that of $\log R_{n-1}$.

The second question is just what is happening to produce the patterns of correlation. Recall that we were led to study this correlation primarily because the instructions to preserve subjective intensity ratios in the responses suggest that $\log R_{n}$ should be highly correlated with $\log R_{n-1}$ (the response ratio hypothesis, Luce \& Green, 1974). The triangular pattern suggests that observers comply with this instruction only when the signals are close together; however, the existence of observer C.K. proves that at least one person can fulfill the instructions reasonably well, independent of signal separation. And that leads us to wonder just how labile the triangle is. Is it possible that, under appropriate instructions, observers can alter their behavior to comply fully with the ratio instructions? If so, and this is now under investigation, it appears that observers are using the attention band in an incidental way to decide when to comply with the ratio instructions. We believe that observers are generally reluctant to preserve ratios throughout the experiment because to do so results in an enormous drift over time in the numbers assigned to particular signals. Thus, because the observers want to maintain some consistency in their responses, they cannot always preserve ratios. The data suggest that observers elect to break the ratio instructions in order to introduce consistency when there is a large change in successive signals.

\section{EXPERIMENT 2 TWO-FREQUENCY ABSOLUTE IDENTIFICATION WITH RANDOM INTENSITIES}

The goal of the next experiment is to investigate the claim that different groups of fibers contribute to the sample formed by two intensities which differ by more than $20 \mathrm{~dB}$. If one assumes the band can be located in a fixed region, then using intensities more than $20 \mathrm{~dB}$ apart guarantees different sample sizes, which should affect the observer's ability to answer questions about not only intensity, but about frequency as well. Our plan is to study the absolute identification of two slightly different frequencies when their intensities vary over a large range and, consequently, where the sample size cannot be held fixed.

Specifically, let the two frequencies be $f_{1}$ and $f_{2}$. On each trial, either $f_{1}$ or $f_{2}$ is presented, and the observer attempts to identify which. In addition, the intensity of each signal is chosen at random (equally likely) from among eight possible intensities according to the following scheme. The eight levels of intensity, which span the $50-\mathrm{dB}$ range from 40 to $90 \mathrm{~dB}$ SPL, are grouped so that six of them are clustered in 2-dB steps over a 10-dB range and the remaining two span the rest of the $50-\mathrm{dB}$ range. The location of the cluster, either at the bottom, middle, or top of the range, defines the three experimental conditions. The scheme is diagramed in Figure 5.

If an observer is free to locate a $10-20-\mathrm{dB}$ attention band wherever he chooses, if that location affects by an order of magnitude the sample size on which frequency identifications are based, and if he is sensitive to the payoff differences resulting from the use of the two different sample sizes, then it is clearly optimal to locate the band so as to span the closely packed cluster of stimulus intensities, wherever that may occur. Such behavior will be 


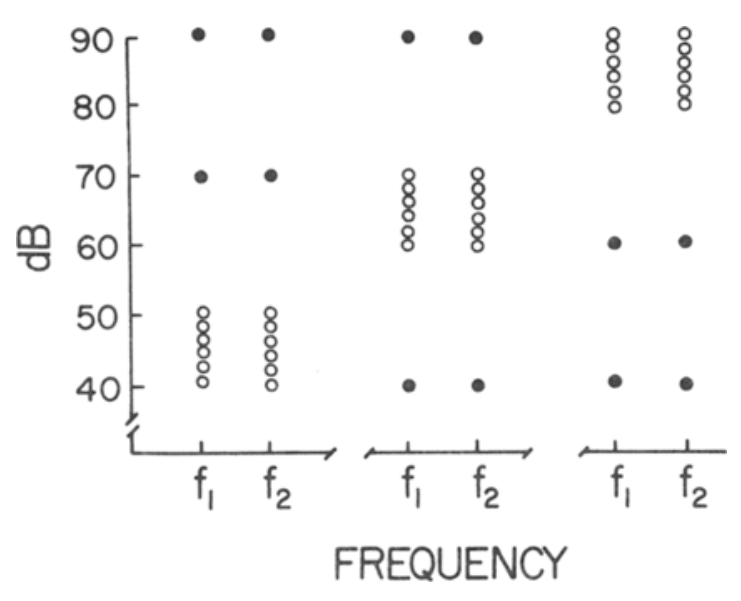

Figure 5. Stimulus sets used in the three conditions of Experiment 2. The open points represent clusters of intensities spaced $2 \mathrm{~dB}$ apart. The panels represent, left to right, low-, medium-, and high-intensity clusters.

evidenced by a higher percentage of correct identifications for the intensities within the cluster than for the other, outlying intensities. In fact, if sample sizes do differ by a factor of 10 , a simple calculation based on normally distributed representations of the signal shows that we should be able to select a frequency pair so that the outlying intensities are at about $60 \%$ correct when those within the cluster are at about $80 \%$ correct. Moreover, a particular intensity will exhibit both values, the lower one when it is in a condition for which it is an outlier and the higher one when it is in a condition for which it is in a cluster.

\section{Procedure}

Signats, which were pulsed sinusoids of $50-\mathrm{msec}$ duration (sinezero on and off), were presented binaurally, in quiet, via TDH-39 headphones, to observers who were tested in double-walled soundtreated chambers (IAC-1200 A). The observer pushed one of two buttons to indicate a response; after the response was recorded, feedback was presented and the next trial began. The trials were self-paced. The two frequencies were $1,000 \mathrm{~Hz}$ and $1,000+\Delta \mathrm{f} \mathrm{Hz}$, where $\Delta \mathrm{f}$ was $8 \mathrm{~Hz}$ for C.B., J.D., and A.G., and $10 \mathrm{~Hz}$ for A.N. and N.R. The intensity of the signal was selected at random from one of three patterns of intensities as described above. The intensities in decibels SPL were $40,42,44,46,48,50,70$, and 90 in Condition I; 40,60,62, 64, 66, 68, 70, and 90 in Condition II; and 40,60,80,82, 84, 86, 88, and 90 in Condition III. It was pointed out to the observers that in each condition six of the intensities lie within a $10-\mathrm{dB}$ range; however, they were instructed not to respond to the intensity of the signal but to try to maximize the percentage of correct responses with respect to frequency identification. Each run of 100 trials began with an ordered presentation of all 16 possible signals. Each observer received considerable training, at least 2,400 trials over a 2 or 3 day period, before any data reported here were taken. This was necessary because randomizing the intensity makes frequency discrimination difficult. Performance was monitored and the experiment began after no noticeable improvement was seen in the course of a day in each of the three conditions, observers C.B., J.D., and A.G. ran 1,200 observations, that is, each point in Figure 6 is estimated from about 150 observations. Observers A.N. and N.R. ran a total of about 2,000 observations, for about $250 /$ point.

\section{Results and Discussion}

The percentages of correct frequency identifications as a function of intensity for the three experimental conditions and five observers are shown in Figure 6. A vertical bar is drawn from the value of each outlier to the mean value of the six intensities within the cluster for that condition. Were the data exactly as predicted, all of these bars should span about 20 percentage points.

One observer, J.D., conforms almost perfectly to the prediction. A second one, C.B., is at the opposite extreme and appears quite unsupportive of the theory; however, it should be noted that he was operating at an overall level of nearly $90 \%$ correct, so his motive for attending to the cluster was slight. The remaining three observers all exhibit the prediction to a degree, especially when the cluster is in the middle of the range. There is, however, some attenuation of

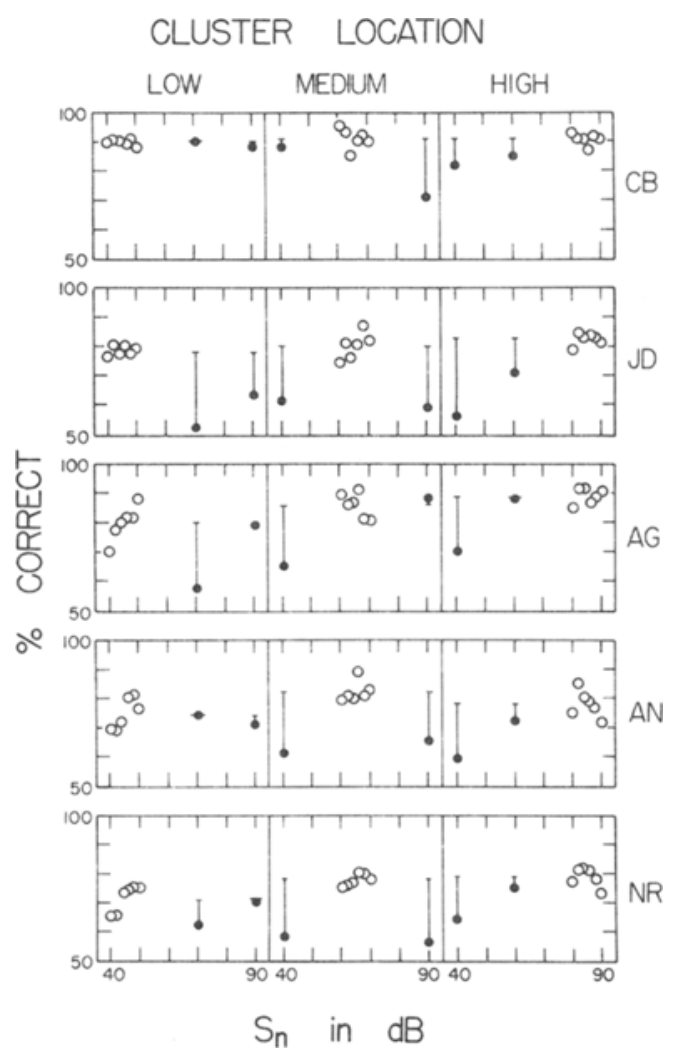

Figure 6. The percentage of correct responses (naming the correct frequency $f_{1}$ or $f_{2}$ ) as a function of the intensity of the stimulus. The panels, left to right, represent the different positions of the clusters; the rows are for individual observers. 
the phenomenon when the cluster is at either extreme. When it is at the upper extreme, all three observers show a smaller drop for the near $(60-\mathrm{dB})$ outlier than for the distant $(40-\mathrm{dB})$ one. When the cluster is at the lower extreme, one of the three observers, A.N., shows no deterioration at all, and the other two, A.G. and N.R., show a drop for the near (60-dB) outlier and none for the distant $(90-\mathrm{dB})$ one. We suspect that the latter discrepancy arises, at least in part, from a widespread tendency of observers to locate the band some of the time at the high-intensity region, even when that is not optimal. The former could occur if the band is a great deal wider at high intensities than at more moderate levels.

The evidence is fairly clear that the observed phenomenon is not simply stimulus-determined. Note that the 40-, 60-, and 90-dB signals each appear both in a cluster and as an outlier. Thus, if the theory were completely correct, each would exhibit both high and low percentages of correct frequency identifications. Table 1 shows that in 11 out of 15 possible cases they do.

Figure 7 presents an analysis of the percentage of correct response conditional on whether two successive signals are in (I) or out $(\mathrm{O})$ of the cluster. Four cases can arise, and if the attention band is located on the cluster, then Condition II should show a high percentage correct, as it does. Even if the last signal were out, the present signal would be in and we expect the condition OI to have a higher percentage than either of the remaining conditions where the present signal is outside the band.

\section{CONCLUSIONS}

The data only partially confirm the existence of an attention band. Our options appear to be to search for a rather different explanation or to assume that our method provides very imperfect control over its location. To date, we are inclined to the latter option and we continue to search for better methods of controlling it. Moreover, if this option is accepted, the data establish both that the sample size can be affected by either large uncertainties in the intensity or frequency of the signal and that the sample size

Table 1

\begin{tabular}{cccc}
\hline Subject & 40 & 60 & 90 \\
\hline C.B. & $\mathrm{X}$ & $\mathrm{H}$ & $\mathrm{X}$ \\
J.D. & $\mathrm{X}$ & $\mathrm{X}$ & $\mathrm{X}$ \\
A.G. & $\mathrm{L}$ & $\mathrm{X}$ & $\mathrm{H}$ \\
A.N. & $\mathrm{X}$ & $\mathrm{X}$ & $\mathrm{X}$ \\
N.R. & $\mathrm{L}$ & $\mathrm{X}$ & $\mathrm{X}$ \\
\hline
\end{tabular}

Note $-X$ denotes cases where both high and low percentage of correct identifications occurred, $L$ where only low ones did, and $H$ where only high ones did.

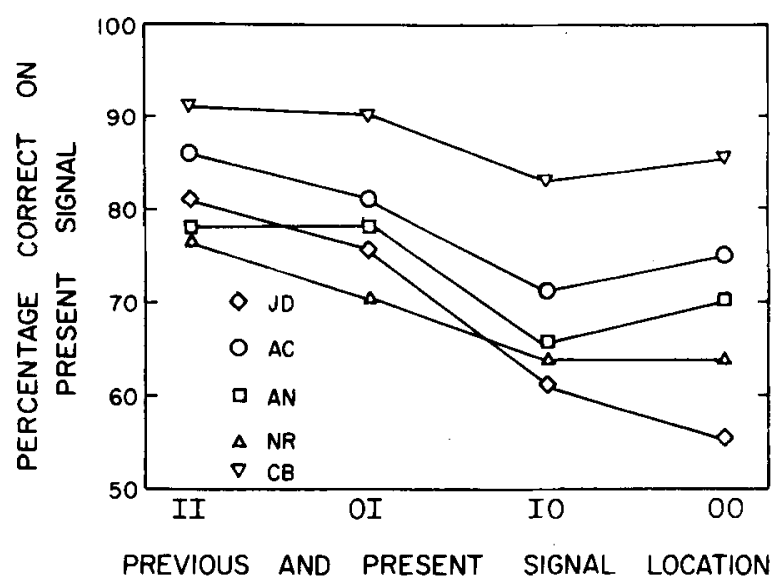

Figure 7. Percentage of correct responses in Experiment 2 as a function of whether or not the previous and present signals were in the intensity cluster. The symbol I means in the cluster and $O$ means outside it, and so OI means that the previous signal is outside the cluster and the present signal is inside.

differentially affects judgments about both dimensions of the signal. This is consistent with the view that the intensity attention band and the frequency critical band are simply two faces of the same process, namely, differential monitoring of groups of neural fibers.

It is perhaps worth noting, in this connection, that the design of the second experiment is, with the roles of frequency and intensity interchanged, suited to studying critical bands. Moreover, this design, while quite tedious, seems potentially adequate to study the width of the band as a function of its domain, either intensity or frequency. One would simply run a number of conditions in which the width of the stimulus cluster (and perhaps the number of levels within the cluster) is varied at each level. The width at which the performance at the edges of the cluster first deteriorates is the width of the band at that level. One difficulty in carrying out such a parametric study arises from the rounding effect which will result from variability in the location of the attention band. Another difficulty is that the relatively small deterioration in discrimination performance would make an estimation of the edge difficult.

Finally, we tentatively suggest that the correlation patterns result because the observers attempt to make the ratio of successive responses equal to the loudness of successive signals, as they are instructed to do. This ratio behavior is most closely followed when successive stimuli differ little in intensity and hence the second signal probably lies in the attention band which was centered on the fibers activated by the previous signal. When large changes of intensity occur, the observer gives an absolute response, one consistent with past responses. This effectively stops 
any drift in the responses which would result if only ratio judgments were given and so it maintains some overall consistency in the judgments. We also showed that the pattern of variability seen in the plot of the coefficient of variation vs. stimulus difference can be deduced from the pattern of correlations as a function of stimulus difference.

\section{REFERENCES}

BaIrd. J. C. Psychophysical analysis of visual space. Oxford: Pergamon, 1970.

BERLINER, J. E., \& DURLACH, N. I. Intensity perception IV. Resolution in roving-level discrimination. Journal of the Acoustical Society of America, 1973, 53, 1270-1287.

BraldA, L. D.. \& DURLACH, N. I. Intensity perception II. Resolution in a one-interval paradigm. Jourmal of the Acoustical Society of America, 1972, 51, 483-502.

Evans, E. F. Cochlear nerve and cochlear nucleus. In W. D. Keidel \& W. D. Neff (Eds.), Handbook of sensory physiology. Auditory system (Vol, V/2). Berlin: Springer Verlag, 1975.

Galambos, R., \& Davis, $H$. The response of single auditory nerve fibers to acoustic stimulation. Journal of Neurophysiology, 1943, 6, 39-57.

GARNER, W. R. An informational analysis of absolute judgments of loudness. Journal of Experimental Psychology, 1953, 46. 373-380.

Green, D. M., \& Luce, R. D. Variability of magnitude estimates: A timing theory analysis. Perception \& Psychophysics, 1974, 15, 291-300.

Green, D. M., Luce, R. D., \& Duncan, J. E. Variability and sequential effects in magnitude production and estimation of auditory intensity. Perception \& Psychophysics, 1977, 22, 450-456.

Jesteadt, W., Luce, R. D., \& Green, D. M. Sequential effects in judgments of loudsess. Joumal of Experimental Psychology, 1977. 3, 92-104.

KiANG, N. Y.S. Discharge patterns of single fibers in the cat's auditory nerve. Research Monograph 35. Cambridge, Mass: MIT Press, 1965, 154 pp.

KIANG, N. Y.-S. A survey of recent developments in the study of auditory physiology. Annals of Otology, Rhinology, and Laryngology, $1968,77,656-676$.

LuCE, R. D., \& GREEN, D. M. The response-ratio hypothesis for magnitude estimation. Joumal of Mathematical Psychology, $1974,11,1-14$

LuCE, R. D., Green, D. M., \& Weber, D. L. Attention bands in absolute identification. Perception \& Psychophysics, 1976, 20. 49-54.
Miller, G. A. The magical number seven, plus or minus two: Some limits on our capacity for processing information. Psychological Review, 1956, 63, 81-97.

Rose, J. E., Brugge, J. F., Anderson, D. J., \& Hind, J. E. Phase-locked response to low-frequency tones in single auditory nerve fibers of the squirrel monkey. Journal of Neurophysiology, 1967, 30, 769-793.

Weber, D. L., GreEN, D. M., \& LuCE, R. D. Effects of practice and distribution of auditory signals on absolute identification. Perception \& Psychophysics, 1977, 22, 223-231.

\section{NOTE}

1. These correlations were computed in the following way. For each pair of stimuli, $s$ and $s^{\prime}$, consider all pairs of successive trials, $n-1$ and $n$, such that $s$ was presented on trial $n-1$ and $s^{\prime}$ on trial $n$. We calculate the correlation between the logarithm of the response on trial $n, \log R_{n}$, with $\log R_{n-1}$ over all such pairs of trials. Since there are 20 signals, there are, in principle, 400 such correlations; however, we require there to be at least three such pairs of trials before entering a correlation in a cell. Next, we averaged together all correlations for which the change in successive signals is the same, i.e., over sets of cells parallel to the major diagonal of the matrix of correlations. Aside from empty cells, there are 20 correlations for a 0 -dB change, 19 each for $a+2-d B$ and $a-2-d B$ change, $\ldots$, and one each for $a+40-d B$ and $a-40-d B$ change. Because large changes occur less frequently than small ones, we further averaged over the large changes so the reported averages are all based on comparable samples. We used the logarithm of the responses for two reasons: first, the raw distribution of responses at a given signal is rather skewed, and second, the hypothesis that observers are preserving subjective ratios in their responses suggests that this is the correct varjable to correlate. However, the correlation surface obtained by using the raw responses is very similar to that for $\log$ responses.

It is important to realize that we are not computing the correlation of all response pairs corresponding to a given signal difference. Our results for that calculation would be trivial, because the range of the responses depends upon the range of the corresponding signals. For example, with no signal change, the signals span a range of over three orders of magnitude, whereas a $+40-\mathrm{dB}$ change occurs for only one pair, 45 followed by $85 \mathrm{~dB}$. In the former case, the range of the responses forces a correlation of nearly 1 even if each of the individual cell correlations was exactly 0 .

(Received for publication December 12, 1977 ; accepted December 21, 1977.) 